\title{
Swimming against the tide: insights from the ORBITA trial
}

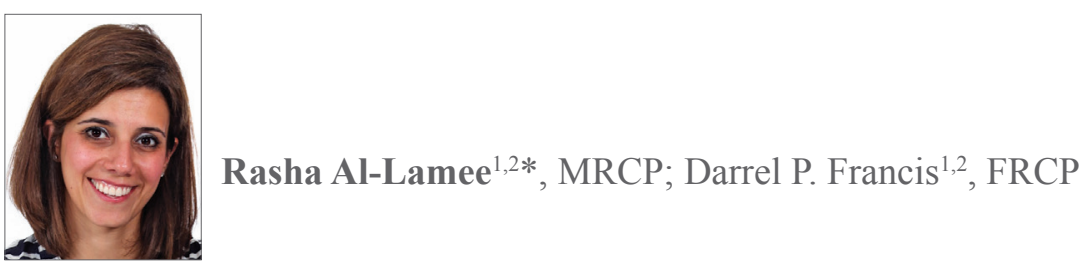

1. Imperial College London, London, United Kingdom; 2. Imperial College Healthcare NHS Trust, London, United Kingdom

All science builds on the science that has been done before. In the case of the placebo-controlled assessment of the efficacy of modern stenting in angina relief, the science that had been done before was non-existent.

Through the retrospectroscope, critics can easily suggest modifications that might have made the effect size larger, but readers will recognise that the investigators had to plan the trial without knowing its results. In the four years of conception, design, execution and completion of the Objective Randomised Blinded Investigation with optimal medical Therapy of Angioplasty in stable angina trial (ORBITA), we fell into the trap of expecting the data to speak for itself ${ }^{1}$. We failed to anticipate the number of voices that would drown out ORBITA with competing choruses of overextrapolation and denigration.

ORBITA was conceived to deliver simple evidence for what we all believed was true, namely that patients with lesions like those in the ORBITA appendix and sufficient angina on medical therapy would indeed get a large benefit from percutaneous coronary intervention (PCI).

ORBITA was designed to expose no more patients (and for no more time) to placebo than necessary. The placebo arm needed to be sufficiently acceptable to patients and their clinicians to prevent undue selection bias and high rates of crossover or dropout. In COURAGE,
$32 \%$ crossed over from the medical therapy arm to the PCI $\mathrm{arm}^{2}$. In FAME 2, the crossovers (with neither myocardial infarction nor ECG changes) comprised the majority of events, and indeed almost all occurred soon after the physician had explained that there was a haemodynamically significant lesion that had not been stented ${ }^{3}$.

The primary endpoint of ORBITA mirrored US Food and Drug Administration and European Medicines Agency recommendations for anti-anginal medications, i.e., treadmill exercise time ${ }^{4,5}$. The exercise protocol was based on the modified Bruce protocol, which is standard for anti-anginal therapies ${ }^{4,6}$.

The sample size was designed to be correct to address the question. Plain balloon angioplasty provided a +96 -second exercise time improvement in the unblinded ACME trial ${ }^{6}$. ORBITA was designed to detect a 30 -second effect size beyond placebo and needed $\sim 200$ patients. Readers will know that the sample size required falls with the square of the effect size one is trying to detect. To design a trial to detect 90 seconds (threefold larger) would need $3^{2}$-fold fewer, i.e., only $\sim 24$ patients. The only reason to carry out a larger study would have been the belief that a smaller effect size than 30 seconds was clinically relevant. To detect a 15 -second effect size (similar to the point estimate of ORBITA) would need $2^{2}$ (fourfold) more patients, i.e., a trial size of $\sim 800$ patients. Randomising the additional 300 patients 
to placebo would only be justified if we would be prepared to recommend PCI for an average benefit of 15 seconds.

Ninety-four percent (94\%) of ORBITA patients had evidence of ischaemia on one or more non-invasive or invasive tests. However, this was not an entry requirement because the majority of PCI worldwide is based on symptoms and angiographically evidenced disease, with approximately $90 \%$ of PCI performed without invasive pressure measurements ${ }^{7}$. Enrolment to ORBITA was designed to be representative of appropriate single-vessel elective $\mathrm{PCI}^{8}$.

Patients and their clinicians were only asked to remain blinded to treatment allocation for six weeks. Forty years of experience of PCI for stable angina and the previously reported dramatic symptom relief meant that it seemed unethical to ask patients to commit to a longer time period in this first blinded trial. Additionally, ORBITA needed to mirror clinical practice by including patients with high-grade stenoses in major epicardial vessels. While such patients would be expected to have the largest angina relief from PCI, they may also face greater risk of events. Therefore, a realistic blinded period had to be set in advance, to ensure the inclusion of lesions such as those in ORBITA.

The battle for funding came next. Applications to all major UK funding bodies and device companies were rejected for a variety of reasons. Expert reviewers informed us that:

- this trial is not important

- it does not address a clinically relevant question

- we know the answer to this question - of course PCI works

- investigators and patients will never agree to take part

- this trial cannot be done

Although their evaluation was disappointing, the planning of ORBITA continued. The trial was eventually funded internally by the Foundation for Circulatory Health and the Imperial Biomedical Research Centre with pressure wires donated by Philips Volcano.

Our fellow interventionists often marvel that we obtained ethical approval. In fact, the appraisal by the national ethics committee was as surprising as that of the funding bodies, but in the opposite direction. The committee raised three queries:

1. "Is it possible that we may be implanting unnecessary stents?"

2. "Does an angioplasty procedure have risks?"

3. "Are there long-term complications following angioplasty?"

When our answer to each of these questions was affirmative, their final question was, "then why has this trial not already been done?". To that, we had no response.

Selection of ORBITA investigators was key to successful conduct. These interventionists had to understand the need for this trial and be driven by curiosity. Their hospitals would make an economic loss on each patient enrolled. They needed to be prepared to enrol all eligible patients including those with severe lesions that may have been excluded from previous trials. We are disappointed to hear accusations of selection bias because of the time taken to enrol 230 patients; in reality, it took time because unquestionably single-vessel coronary disease, with stable angina, is rarer than we had thought.

Recruitment of ORBITA participants required commitment from the investigators. A consultant cardiologist approached patients after the diagnostic angiogram. Explaining the rationale and design of ORBITA took time and dedication. We were fortunate that the majority understood the importance of this trial, and a respectable $63 \%$ of all eligible patients were recruited.

The ORBITA patients were consented to the 50:50 chance of having a placebo procedure. They understood that, if randomised to placebo, this procedure would carry risks, and that they might require another procedure to have clinical PCI after the trial end. They were also willing to measure their heart rate and blood pressure at home, and have telephone consultations several times per week with a consultant cardiologist, to introduce and up-titrate anti-anginal medications. The ORBITA patients showed that, with the commitment of an academic team and participants, placebocontrolled trials of procedures can indeed be done.

The results of ORBITA were a surprise to us. Since the design targeted a conservative expectation of effect size, we expected PCI, with anatomic stenosis resolution, easily to show a statistically significant effect beyond placebo. Checking and rechecking of the data only confirmed the same results and so, despite our preconceptions, we had to concede that the link between treating a severe epicardial stenosis with stenting and symptomatic relief was more complex than we had supposed.

More surprising than the results themselves was the subsequent reaction to them. The lay press, medical "key opinion leaders" and social media commentators competed in the speed and ferocity with which they voiced their opinions. Some overextrapolated the results to undermine PCI as a whole for stable angina. Others were just as quick to undermine the trial with remarks that were a mix of intemperate, innumerate and plain inaccurate.

The reception experienced by ORBITA, and by COURAGE a decade ago, emphasises the polarity of opinions and reflects a need for more such trials. Clinical trialists should be the first to acknowledge the limitations of their research, putting their own results into perspective. Every step in medical research should be seen as exactly that, "one more step forward". The equal servings of praise and ridicule may continue for some time but ORBITA has undoubtedly shown that placebo-controlled interventional trials can be done, do provide new information, and should be as standard for interventional procedures as they are for medication.

\section{Conflict of interest statement}

R. Al-Lamee has received speaker's honoraria from Philips Volcano.

D. Francis has no conflicts of interest to declare.

\section{References}

1. Al-Lamee R, Thompson D, Dehbi HM, Sen S, Tang K, Davies J, Keeble T, Mielewczik M, Kaprielian R, Malik IS, Nijjer SS, Petraco R, Cook C, Ahmad Y, Howard J, Baker C, Sharp A, Gerber R, Talwar S, Assomull R, Mayet J, Wensel R, Collier D, Shun-Shin M, Thom SA, Davies JE, Francis DP; ORBITA investigators. Percutaneous coronary intervention in stable angina (ORBITA): a double-blind, randomised controlled trial. Lancet. 2017 Nov 1. [Epub ahead of print]. 
2. Boden WE, O'Rourke RA, Teo KK, Hartigan PM, Maron DJ, Kostuk WJ, Knudtson M, Dada M, Casperson P, Harris CL, Chaitman BR, Shaw L, Gosselin G, Nawaz S, Title LM, Gau G, Blaustein AS, Booth DC, Bates ER, Spertus JA, Berman DS, Mancini GB, Weintraub WS; COURAGE Trial Research Group. Optimal medical therapy with or without PCI for stable coronary disease. N Engl J Med. 2007;356:1503-16.

3. De Bruyne B, Fearon WF, Pijls NH, Barbato E, Tonino P, Piroth Z, Jagic N, Mobius-Winckler S, Rioufol G, Witt N, Kala P, MacCarthy P, Engström T, Oldroyd K, Mavromatis K, Manoharan G, Verlee P, Frobert O, Curzen N, Johnson JB, Limacher A, Nüesch E, Jüni P; FAME 2 Trial Investigators. Fractional flow reserve-guided PCI for stable coronary artery disease. $N$ Engl J Med. 2014;371: 1208-17.

4. Chaitman BR, Skettino SL, Parker JO, Hanley P, Meluzin J, Kuch J, Pepine CJ, Wang W, Nelson JJ, Hebert DA, Wolff AA; MARISA Investigators. Anti-ischemic effects and long-term survival during ranolazine monotherapy in patients with chronic severe angina. J Am Coll Cardiol. 2004;43:1375-82.

5. Borer JS, Fox K, Jaillon P, Lerebours G; Ivabradine Investigators Group. Antianginal and antiischemic effects of ivabradine, an I(f) inhibitor, in stable angina: a randomized, doubleblind, multicentered, placebo-controlled trial. Circulation. 2003; 107:817-23.

6. Parisi AF, Folland ED, Hartigan P. A comparison of angioplasty with medical therapy in the treatment of single-vessel coronary artery disease. Veterans Affairs ACME Investigators. $N$ Engl $J$ Med. 1992;326:10-6.

7. Gotberg M, Cook CM, Sen S, Nijjer S, Escaned J, Davies JE. The Evolving Future of Instantaneous Wave-Free Ratio and Fractional Flow Reserve. J Am Coll Cardiol. 2017;70:1379-402.

8. Patel MR, Calhoon JH, Dehmer GJ, Grantham JA, Maddox TM, Maron DJ, Smith PK. ACC/AATS/AHA/ASE/ASNC/ SCAI/SCCT/STS 2017 Appropriate Use Criteria for Coronary Revascularization in Patients With Stable Ischemic Heart Disease : A Report of the American College of Cardiology Appropriate Use Criteria Task Force, American Association for Thoracic Surgery, American Heart Association, American Society of Echocardiography, American Society of Nuclear Cardiology, Society for Cardiovascular Angiography and Interventions, Society of Cardiovascular Computed Tomography, and Society of Thoracic Surgeons. J Nucl Cardiol. 2017;24:1759-92. 